\title{
Réfractomètre : référence de longueur d'onde dans l'air
}

\author{
H. Fang, N. Khélifa, K. Etsouri, R. Thibout, V. Levelly et P. Juncar \\ Institut National de Métrologie, BNM-CNAM, 292 rue Saint-Martin, 75141 Paris \\ cedex 03, France
}

\begin{abstract}
Résumé : L'une des principales sources d'incertitudes limitant la précision des mesures dimensionnelles dans l'air par méthode interférométrique est liée à l'incertitude de mesure de l'indice de réfraction et à celle de ses fluctuations. A ce jour les meilleurs réfractomètres n'atteignent que des incertitudes à l'échelle des $10^{-7}$, et leurs temps de réponse relativement grands ne permettent pas d'effectuer des mesures en temps réel. Dans cet article, nous présentons le principe d'un nouveau type de réfractomètre, en développement à l'INM, pour la mesure en temps réel de l'indice de l'air avec une précision d'environ $10^{-9}$, ainsi que quelques résultats préliminaires.
\end{abstract}

\section{PRINCIPE DE FONCTIONNEMENT [1][2][3]}

L'élément de base du réfractomètre est constitué d'un interféromètre Fabry-Perot Plan-Plan en zérodur à double voie dont l'une peut être mise sous vide indépendamment de l'autre (figure 1). Chacune de ses deux cavités est éclairée par une diode laser dont la fréquence est asservie sur un pic de transmission du Fabry-Perot.

Initialement, les deux cavités du F-P sont à l'air, et les deux diodes sont asservies sur le même pic $K$ du F-P avec un battement de fréquence nulle. Ainsi, $\lambda_{1}=\lambda_{2}=\frac{2 \ell}{\kappa}$ et $v_{1}=v_{2}=\kappa \cdot \frac{c}{2 n \ell}$ où $c$ est la vitesse de la lumière dans le vide.

La voie 1 est ensuite mise sous vide, la fréquence des lasers restant asservie sur le même pic du Fabry-Perot. Après pompage, on a toujours $\lambda_{1}^{\prime}=\lambda_{2}=\frac{2 \ell}{K}$. Évidemment $v_{1}^{\prime}$ devient $v_{1}^{\prime}=\kappa \cdot \frac{c}{2 \ell}$. Ceci donne une différence de fréquence $\Delta v^{\prime}=v_{1}^{\prime}-v_{2}$. La fréquence de la diode LD1 est comparée à une fréquence de référence $v_{0}$ par un battement de fréquence $\Delta v^{\prime}=v_{1}^{\prime}-v_{0}$.

Ainsi à partir de la connaissance de $v_{0}$ et des mesures de $\Delta v$ et de $\Delta v^{\prime}$, on déduit la valeur de l'indice de l'air $n=\frac{v_{0}+\Delta v}{\left(v_{0}+\Delta v\right)-\Delta v}$. On constate qu'elle est indépendante de la valeur de $\kappa$ et de la longueur $\ell$ du F-P. De plus, la valeur de la longueur d'onde de la diode laser est donnée par : $\lambda_{2}=\frac{c}{v_{0}+\Delta v^{\prime}}$ donnant lieu à une référence de longueur d'onde dans l'air, 
indépendante de l'indice de réfraction n et de ses éventuelles fluctuations, la cavité F-P se comporte comme une véritable cale étalon optique.

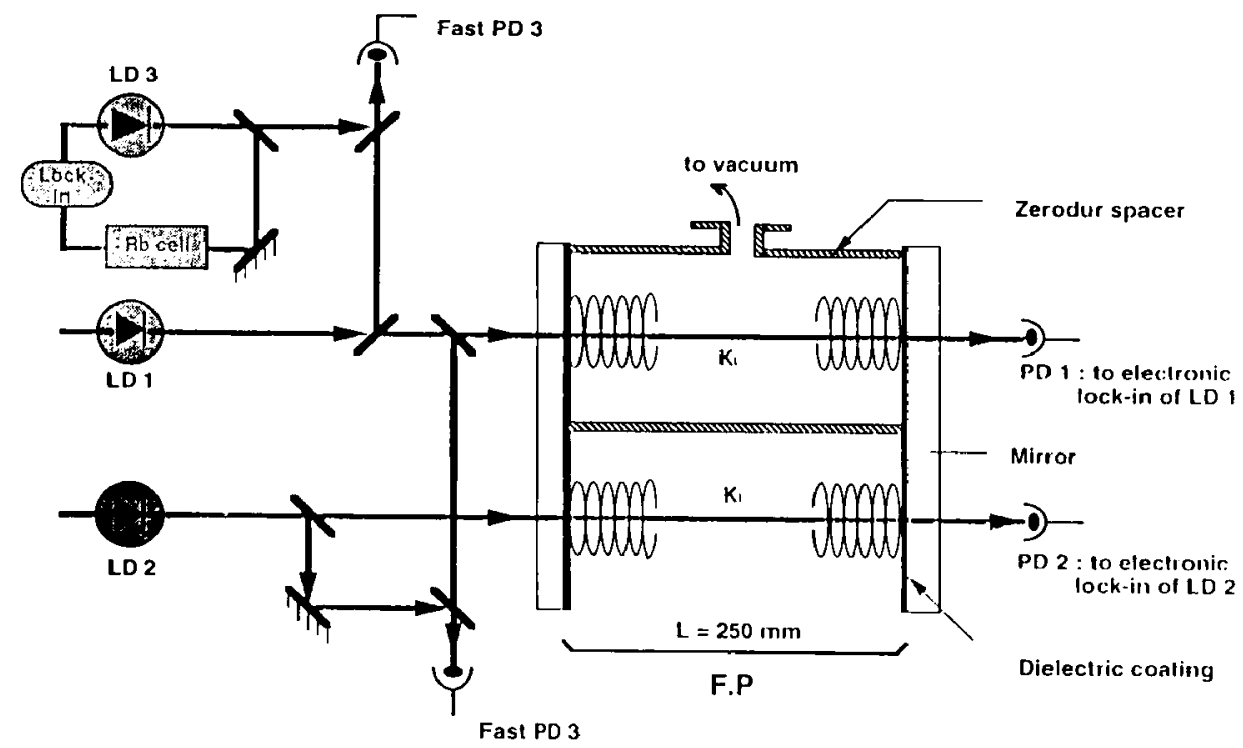

Figure 1 : Réfractomètre de l'INM.

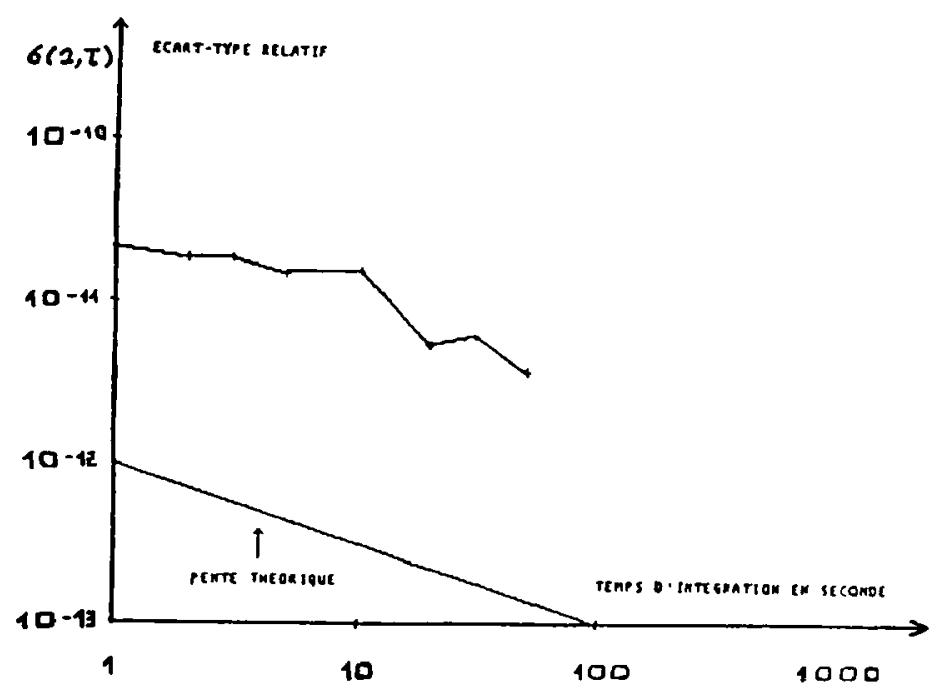

Figure 2 : Stabilité mécanique du F-P. 


\section{QUELQUES RÉSULTATS PRÉLIMINAIRES [2][3]}

\subsection{Balayage de plus de $100 \mathrm{GHz}$ sans saut de mode}

On a pu balayer continûment la fréquence de la diode laser DBR (Distributed Bragg Reflector) LD1 sur une plage de plus de $100 \mathrm{GHz}$; l'asservissement du DBR sur le pic du F-P est assuré par une double boucle contenant une composante lente (jouant sur la température) et une composante rapide (sur le courant).

\subsection{Comportement du F-P à des différences de pression}

Nous avons pu mesurer les effets de compression de l'interféromètre F-P lors du pompage, grâce aux mesures de battement de fréquences entre la référence $v_{0}$ et la diode asservie sur le F-P. Ces effets sont relativement faibles $(<\lambda / 10)$ et n'ont aucune conséquence sur le principe de la méthode.

\subsection{Stabilité mécanique du Fabry-Perot}

La comparaison de la fréquence asservie sur l'un des pics de transmission du Fabry-Perot avec celle asservie sur une raie hyperfine du Rubidium nous a permis de tester la stabilité mécanique du Fabry-Perot. La courbe obtenue, par analyse de la variance d'Allan (figure 2), montre une stabilité de l'ordre de $5.10^{-12}$ sur une centaine de secondes, mettant ainsi en évidence la capacité offerte par cette méthode de fournir une référence de longueur d'onde dans l'air stable.

\section{Références}

[1] Juncar P., Etsouri K. et Khélifa N., "A new refractometer : towards air wavelength reference", 8th IPES, France, 1995.

[2] Fang H., Rapport de mémoire de DEA "Etude et Caractéristiques d'un Réfractomètre", CNAM, juin 1995.

[3] Khélifa N., Fang H., Etsouri K. et Juncar P., " Vers une référence de longueur d'onde dans l'air", Horizons de l'Optique, 1995. 